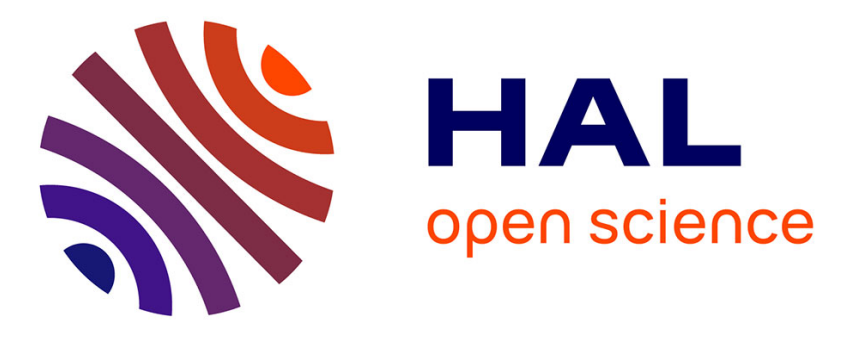

\title{
Actionneur linéaire synchrone à aimants permanents multi-tiges
}

Pierre-Emmanuel Cavarec, Hamid Ben Ahmed, Bernard Multon, Marc

Antunes

\section{- To cite this version:}

Pierre-Emmanuel Cavarec, Hamid Ben Ahmed, Bernard Multon, Marc Antunes. Actionneur linéaire synchrone à aimants permanents multi-tiges. Electrotechnique du Futur, Dec 2003, GIF SUR YVETTE, France. 10p. hal-00674675

\section{HAL Id: hal-00674675 \\ https://hal.science/hal-00674675}

Submitted on 27 Feb 2012

HAL is a multi-disciplinary open access archive for the deposit and dissemination of scientific research documents, whether they are published or not. The documents may come from teaching and research institutions in France or abroad, or from public or private research centers.
L'archive ouverte pluridisciplinaire HAL, est destinée au dépôt et à la diffusion de documents scientifiques de niveau recherche, publiés ou non, émanant des établissements d'enseignement et de recherche français ou étrangers, des laboratoires publics ou privés. 


\title{
Actionneur linéaire synchrone à aimants permanents multi-tiges
}

\author{
P.E. CAVAREC, H. BEN AHMED, B. MULTON, M. ANTUNES
}

\begin{abstract}
Résumé- A contraintes magnétiques et thermiques données, on montre que l'obtention de poussées volumiques élevées nécessite la multiplication des surfaces d'entrefer et l'utilisation de bobinages globaux (ou centralisés). Sur la base de ce concept, un actionneur linéaire multi-tiges a été conçu, étudié et réalisé. Dans cet article, les auteurs décrivent son principe de fonctionnement, les solutions mécaniques et électromagnétiques développées pour sa faisabilité technique ainsi que ses performances théoriques et expérimentales.
\end{abstract}

Mots clés-Actionneur synchrone linéaire, aimants permanents, polyentrefer, force volumique, dynamique mécanique.

\section{INTRODUCTION}

C lassiquement, lorsqu'il s'agit de produire des couples ou des forces élevées, on accroît les nombres de pôles et on réduit le pas polaire pour minimiser la quantité de circuit magnétique mais les limites physiques de pression magnétique tangentielle d'entrefer subsistent. Cette pression est limitée par l'induction d'entrefer et par l'intensité maximale du champ induit. L'induction d'entrefer est limitée par la saturation des matériaux ferromagnétiques et l'intensité du champ par les échauffements ou/et la désaimantation des aimants éventuels. Ainsi, les pressions tangentielles maximales que l'on peut ainsi atteindre dépassent difficilement les $10 \mathrm{~N} / \mathrm{cm}^{2}$.

Dans le cas des systèmes à mouvement linéaire, les entraînements nécessitant une poussée élevée (applications embarquées de type aéronautique, train pendulaire, compensation active des vibrations...) utilisent généralement soit des actionneurs hydrauliques ou pneumatiques soit des actionneurs tournants classiques accouplés à des systèmes mécaniques de transformation de mouvement rotation/translation (ex. vis-écrou).

Ces solutions génèrent notamment :

- des limitations de la bande passante mécanique et/ou de contrôlabilité ;

- des contraintes mécaniques supplémentaires d’usure, de bruits ;

- une limitation du rendement énergétique global ;

SATIE (UMR CNRS 8029) - Antenne de Bretagne, équipe "ActionneursTransport-Energie”, ENS Cachan-Antenne de Bretagne, campus de Ker lann, 35170 Bruz.benahmed@bretagne.ens-cahan.fr
- la nécessité d'une alimentation en fluide sous pression (masse des canalisations, maintenance, coût énergétique...).

Des solutions innovantes de type polyentrefer ont été imaginées en particulier au laboratoire d'électrotechnique d'Orsay [1]. Ces solutions, combinant la multiplication des surfaces d'entrefers et l'emplois d'un bobinage global, permettent l'obtention de performances volumiques très élevées [2] pourvus que la complexité mécanique soit accompagnée de solutions satisfaisantes sur les plans économique et de faisabilité. Les différentes architectures polyentrefers à bobinages globaux (la distinction est importante avec les autres) développées à ce jour essentiellement au laboratoire d'Orsay, au SATIE (depuis les années 90) et au LEM ont mis en évidence le fort potentiel de ce principe mais également les nombreuses difficultés de sa mise en œuvre.

L'architecture décrite dans cet article tente d'apporter, dès la conception, des solutions notamment mécaniques à ces difficultés. L'objet de cette communication est donc de montrer l'intérêt de la multiplication des surfaces d'entrefer à travers l'étude théorique et expérimentale d'un actionneur linéaire multi-tiges.

\section{ETAT DE L'ART SUR LES ACTIONNEURS POLYENTREFERS}

Parmi les topologies électromagnétiques non conventionnelles, les actionneurs polyentrefers tiennent probablement la place la plus innovante. L'objectif est d'augmenter les performances (force ou couple) des actionneurs de grandes dimensions (dimension caractéristique supérieure à la dizaine de centimètres), principalement destinés aux entraînements directs [3]. Pour cela, l’idée de base est de remplir au mieux le volume disponible, afin d'augmenter ses performances volumiques. Un actionneur est alors constitué de plusieurs modules de base, plus ou moins indépendants, ayant chacun leur propre zone active d'entrefer. On augmente, de cette façon, la surface d'entrefer dans un volume donné. En considérant que la pression électromagnétique tangentielle peut être sensiblement maintenue, les performances globales en effort augmentent.

Sur cette idée, on retrouve deux voies distinctes.

\section{A. Solutions polyentrefers multi-moteurs}

La première, et la plus connue, bien qu'encore industriellement très marginale, consiste à assembler des 
moteurs indépendants les uns des autres. On obtient alors des structures que nous appellerons polyentrefers multi-moteurs, ayant un couplage polaire (i.e. couplage "classique» des machines à champs tournants). Les principales réalisations de ce type de structures sont des actionneurs polydiscoïdes, dont de nombreux développements industriels existent [4]. Il s'agit généralement d'une association d'actionneurs discoïdes à aimants permanents, à grand nombre de pôles et à champ tournant (figure 1). Les aimants sont situés sur les disques rotoriques. Le stator porte les bobinages de puissance triphasés. Ces différents actionneurs sont mécaniquement reliés entre eux par l'arbre de rotation central.

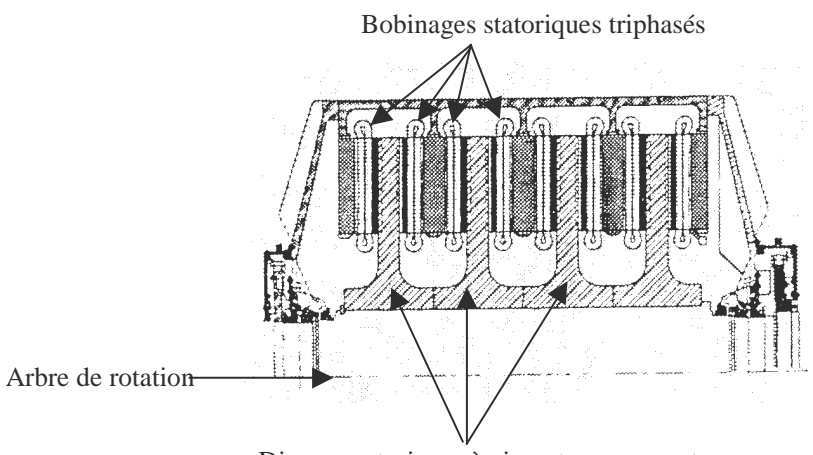

Disques rotoriques à aimants permanents

Fig. 1 Actionneur polydiscoïde Jeumont Industrie [4]

L'intérêt des architectures à champ axial (discoïdes) réside dans la facilité de leur association en cascade. En effet, lorsqu'on accroît le nombre de pôles d'une machine à champ axial elle devient extra-plate. Pour occuper au mieux un volume cylindrique disponible, il est alors aisé d'en associer plusieurs en "série " sur le même arbre [5]. Dans le cas d'architectures à champ radial, plus conventionnelles, l'accroissement de la polarité conduit à des structures en couronne, donc creuses. La bonne occupation de l'espace disponible nécessiterait la réalisation d'architectures polycylindriques qui, bien qu'elles aient déjà été envisagées [6], semblent trop complexes à mettre en œuvre sur le plan mécanique.

Par ailleurs, nous trouvons dans [7], [8] et [9] des configuration multi-moteurs de type linéaire.

\section{B. Les actionneurs polyentrefers à bobinages globaux}

La seconde voie a été développée, au début des années 80, au laboratoire d'électrotechnique d'Orsay des universités Paris VI et XI suite aux travaux de M. le professeur C. Rioux [1]. Ces travaux ont été ensuite, à partir des années fin 80, poursuivies au SATIE (ex. Lesir) ainsi qu'au laboratoire LEM de l'université de Montpellier. L’idée est toujours d'augmenter les surfaces d'entrefer dans un volume donné, mais en exploitant les avantages d'un bobinage de puissance global, commun, pour une même phase, à toutes ces surfaces. Rappelons que cette architecture permet de découpler les échelles locales (correspondant à la génération du couple) et l'échelle globale liée au bobinage. Ceci permet d'engendrer des efforts par unité de volume importants supérieurs à ceux obtenus à partir d'une disposition « classique » à bobinage répartis [10], [27], [28].
1) Les actionneurs polyentrefers à réluctance variable La première réalisation conçue par MM. Rioux et Lucidarme (1980), est une machine triphasée polydiscoïdes à réluctance variable pure, dont la puissance est de $200 \mathrm{~kW}$ à 8 tr/s [11].

Il faut néanmoins noter qu'une autre réalisation, sur le même principe, avait été présentée auparavant par Lewis E.Unnewhr de la compagnie Ford [12].

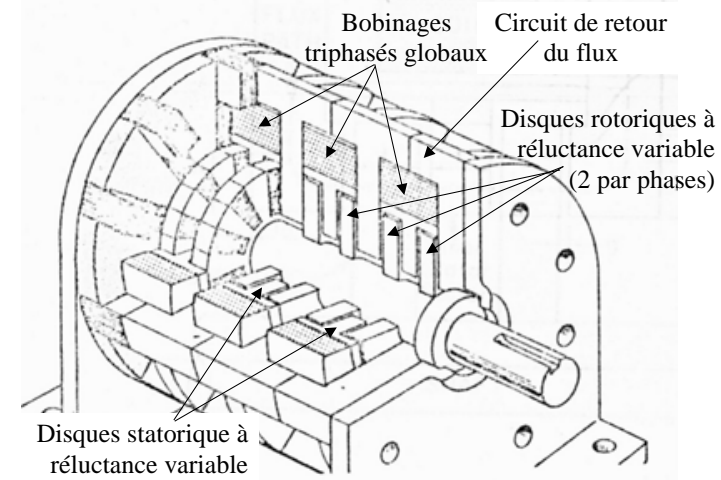

Fig. 2 Machine à réluctance variable discoïde polyentrefer à bobinages globaux [12].

Le prototype réalisé était monophasé, d'une puissance de 6 $\mathrm{kW}$ à $5000 \mathrm{tr} / \mathrm{mn}$ et comportait deux disques de 17 plots, soit une fréquence maximale de $1,4 \mathrm{kHz}$.

Plus tard, un actionneur poly-cylindrique (figure 3) a été étudié au Laboratoire d’Orsay [6]. Il présente deux particularités :

- Il s’agit d’un actionneur à excitation bobinée homopolaire, basé sur les mêmes motifs réluctants ;

- Le couplage est dentaire Vernier (répartition du bobinage dans des encoches).

Son couple massique est cependant resté faible (200Nm pour $100 \mathrm{~kg}$ de masse active).

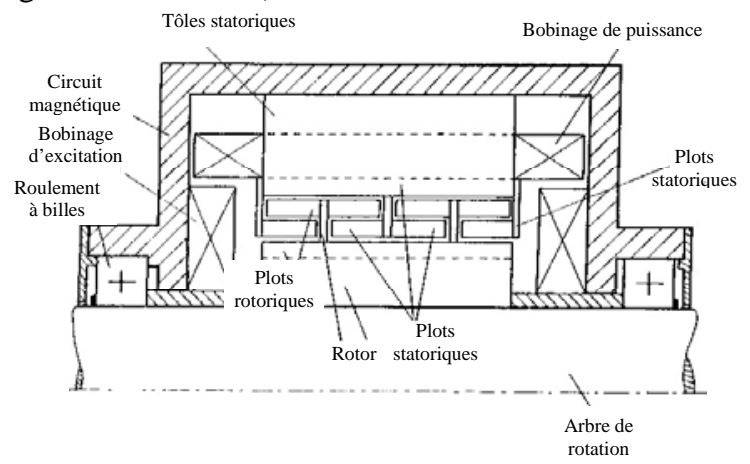

Fig. 3 Machines polycylindriques à couplage dentaire Vernier à excitation bobinée homopolaire [6].

La quatrième machine à réluctance variable est à champ longitudinal [13] (figure 4). Elle a été étudiée par MM. Matt, Rioux et Lucidarme (1987). Il s'agit d'un actionneur linéaire à plaques. Elle possède 26 entrefers d'environ 2/100 ème d'épaisseur (en réalité frottants). La partie active est constituée de 14 plaques fixes et de 13 plaques mobiles de $1 \mathrm{~mm}$ d'épaisseur. La course vaut $40 \mathrm{~mm}$ et le pas dentaire est de $6 \mathrm{~mm}$. Les performances, inégalées à ce jour, sont en régime 
permanent de $1200 \mathrm{~N}$ avec une masse active d'environ $2 \mathrm{~kg}$ [6] [14].

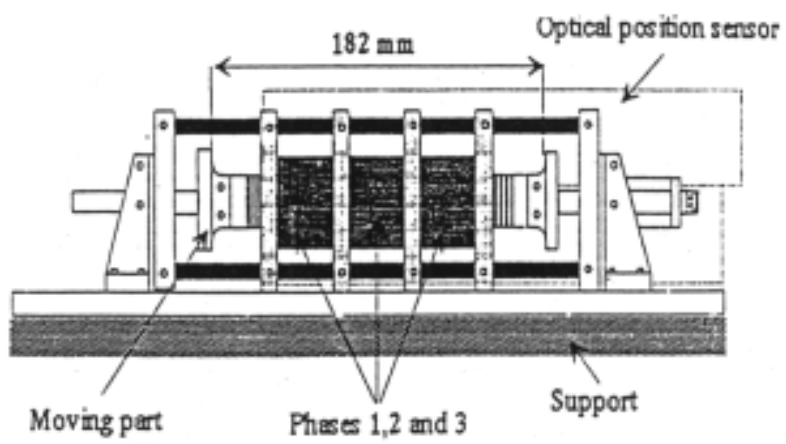

Fig. 4 Actionneur linéaire à réluctance variable à plaques mobiles [6], [13] [14].

\section{2) Les actionneurs à aimants permanents}

En ce qui concerne les actionneurs à aimants permanents, on peut établire suivant des considérations simples quatre motifs élémentaires [15] donnés à la figure 5.
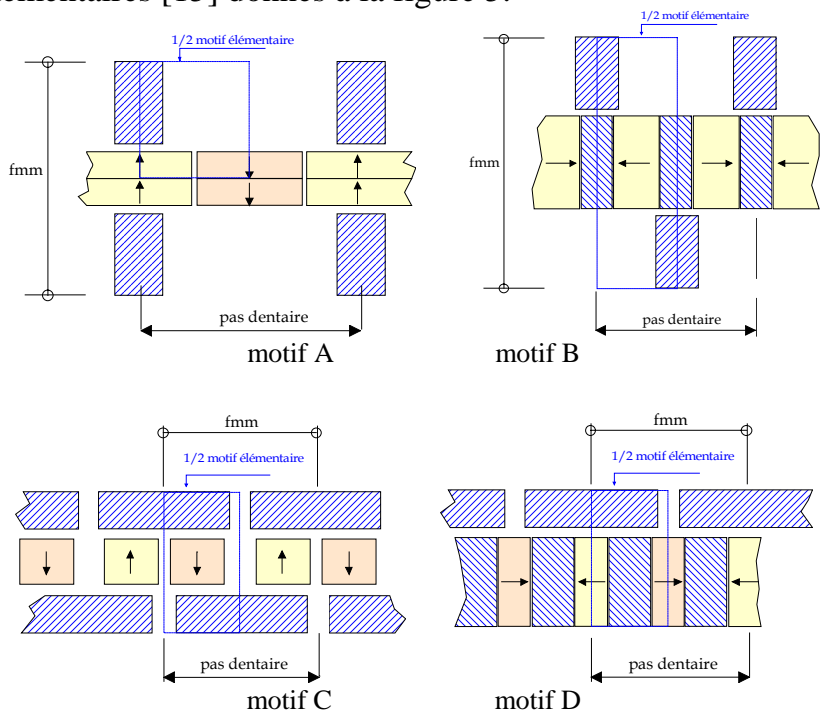

Fig. 5 Motifs élémentaires fondamentaux [15].

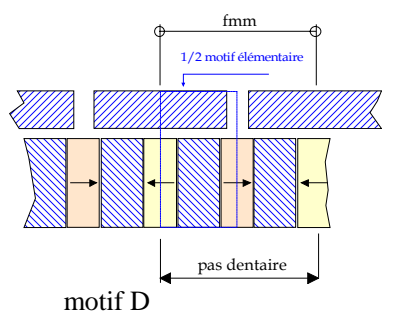

Dans ces quatre motifs de bases, la partie mobile peut être indifféremment la partie réluctante ou la partie aimantée.

Ces motifs se sont retrouvés dans différents actionneurs réalisés au cours des années fin 1980-2000, nous en présentons quelques uns.

La première application polyentrefer à aimants permanents voit le jour en 1987. C'est une machine à disques à aimants alternés (motif A) conçue avec la collaboration de CegelecNancy (figure 6). Elle a été dimensionnée pour l'entraînement d'un bras de robot pour le compte du C.E.A. [16], [17]. Plusieurs prototypes ont été réalisés. On peut citer en particulier le prototype bi-rotors développant un couple permanent de 330 N.m pour une vitesse de rotation de 120 $\mathrm{tr} / \mathrm{min}$ et une masse totale de $40 \mathrm{~kg}$.

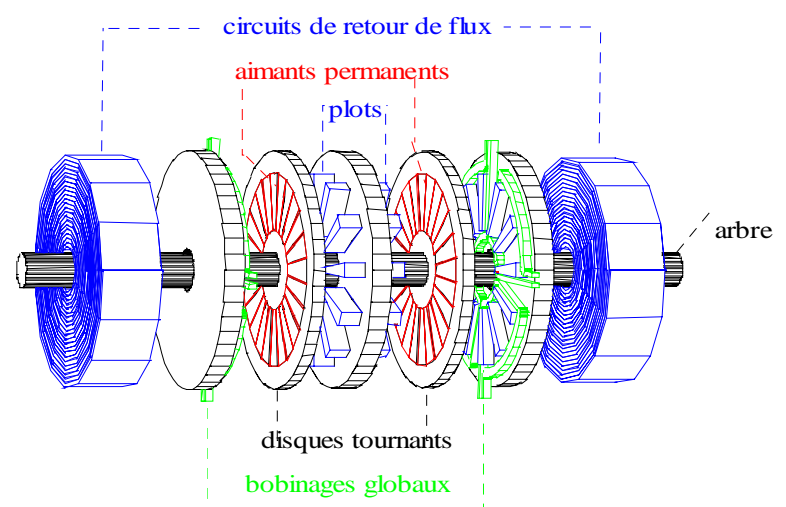

Fig. 6 Moteur à disques à aimants alternés Cegelec-Nancy [15].

Le motif $\mathrm{B}$ a été appliqué sur un prototype étudié au L.E.M. avec les travaux de P.Poulot et P. Tia [18]. Cette structure (figure 7) ne possède qu'un disque mobile (c'est-à-dire deux surfaces d'entrefers). Les trois phases sont placées côte à côte. Il s'agit d'une machine à bobinages globaux mais non polyentrefers, au même titre que les moteurs pas à pas à rotor disque de Portescap.

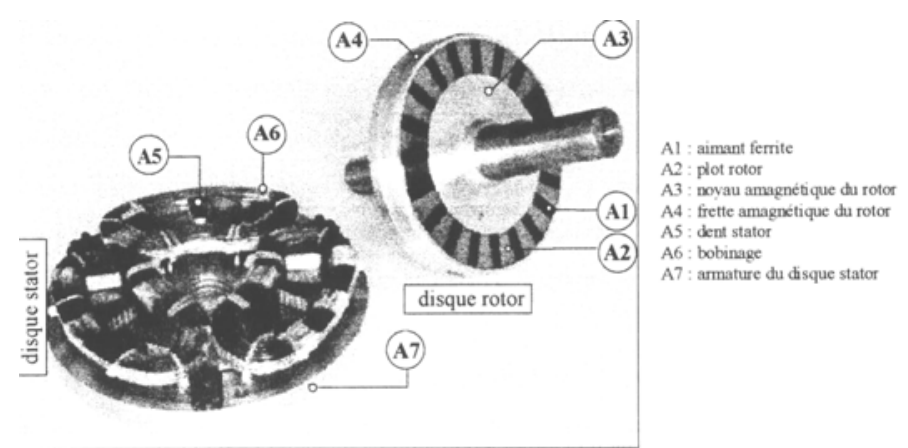

Fig. 7 Actionneur polyphasé à aimants monodiscoïde du LEM [18].

Enfin l'un des derniers actionneurs en date, réalisé au SATIE en collaboration avec la société Radio Energie et la D.R.E.T., utilise le motif C [19], [20]. C'est un actionneur linéaire à aimants alternés, dont les structures de plots sont en étoiles disposées en quinconce et imbriquées (figure 8). Il possède 36 surfaces d'entrefer, sa course est de $55 \mathrm{~mm}$ pour un pas dentaire de $13 \mathrm{~mm}$. Le diamètre extérieur, hors tout, est de $210 \mathrm{~mm}$, et sa longueur active est de $280 \mathrm{~mm}$. En régime impulsionnel, l'effort crête est estimé à $20 \mathrm{kN}$. Une seconde maquette multi-plaques [21] a été également étudiée et réalisée dans le même contexte industriel (voir figure 9). La maquette réalisée possède 16 surfaces d'entrefer plan et développe une poussée de $20 \mathrm{kN}$ pour une masse totale (y compris le système de refroidissement des bobinages) de 850 $\mathrm{kg}$.
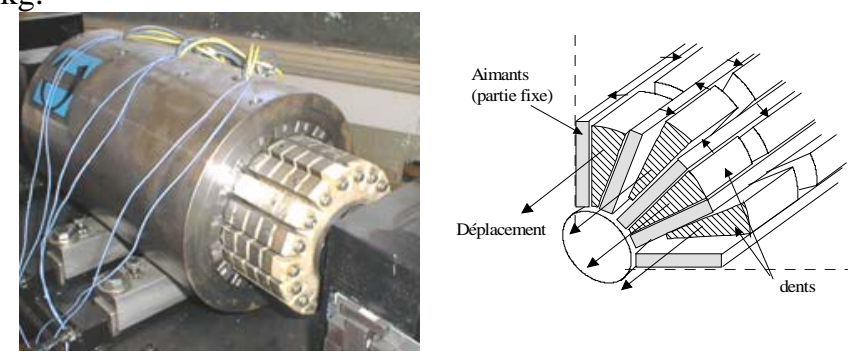

Fig. 8 Actionneur linéaire polyentrefer à structure étoile [19], [20]. 


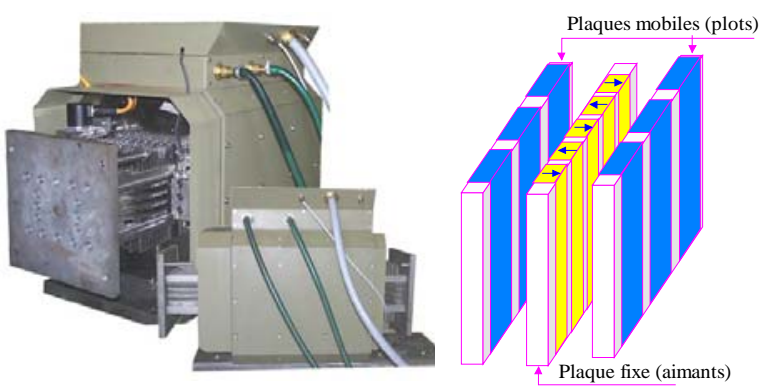

Fig. 9 Actionneur linéaire polyentrefers à structure plaques [21].

Toutes ces réalisations polyentrefers à bobinage global ont permis de mettre en évidence l'intérêt de la multiplication des surfaces d'entrefers dans une configuration à bobinage global ou centralisé. Néanmoins, leurs performances ont été souvent limitées par les difficultés mécaniques en particulier de guidage et de réalisation. Suite à ce constat, nous avons entamé des travaux de conception d'un actionneur linéaire polyentrefers en tentant d'y intégrer dès le départ les contraintes mécaniques pré-citées et en essayant d'y apporter des solutions viables. Cette recherche nous a menés à une solution dite multi-tiges utilisant comme base le motif $\mathrm{D}$ (figure 10) [22], [23], [24]. Cet article en donne le principe de fonctionnement, les solutions mécaniques développées ainsi que quelques performances théoriques et expérimentales obtenues.

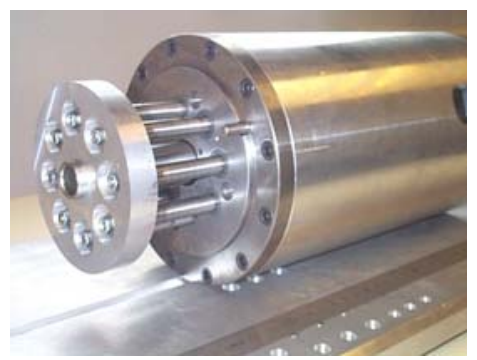

Fig. 10 Actionneur linéaire polyentrefer à structure tiges

\section{ACTIONNEUR MULTI-TIGES}

\section{A. Description et principe de fonctionnement}

Il s'agit d'un actionneur synchrone à aimants permanents, à réluctance localement variable à champ longitudinal et à bobinage global (motif D). La structure d'actionneur proposée combine les aspects fondamentaux suivants :

- La partie mobile, composée de plusieurs tiges cylindriques, est passive ;

- La partie fixe comporte aussi bien l'induit (puissance) que l'inducteur (excitation par aimants permanents) ;

- L'induit triphasé entoure totalement la partie mobile (architecture à bobinages globaux) ;

- La transmission de la force s'effectue de telle sorte que les parties mobile ne soient sollicitées qu'en traction ;

Une vue schématique éclatée de l'actionneur (sans les capteurs) est donnée à la figure 11.

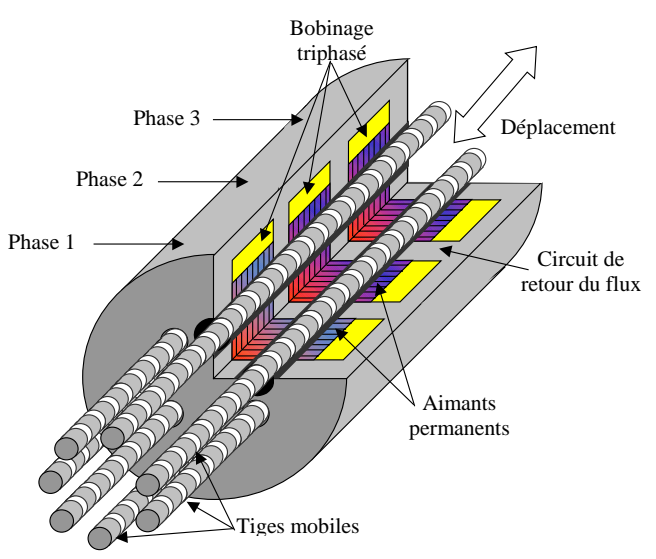

Fig. 11 Vue schématique de l'actionneur

Les deux positions caractéristiques de conjonction positive et négative sont représentées ci-dessous (figure 12) montrant ainsi la variation du flux inducteur (aimants) par rapport à la position de la partie mobile (tiges).

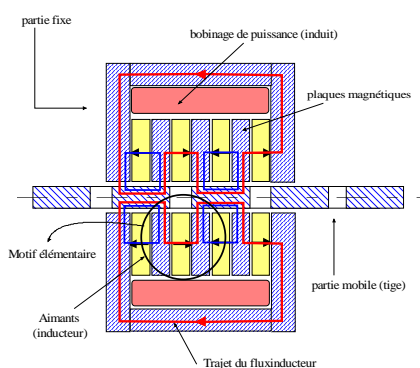

(a) Conjonction « positive "

Fig. 12 Les deux Positions de conjonctions de l'actionneur (une seule phase est représentée)

\section{B. Guidages mécaniques glissants}

L'effort normal à la surface d'entrefer (effort parasite) augmente avec le décentrage de la partie mobile avec la partie fixe (figure 13a). Outre la réduction de sa valeur par une optimisation des dimensions du motif élémentaire, il peut être intéressant également de limiter autant que possible ce décentrage.

Avec des méthodes de guidage ponctuels classique, limiter le décentrage en maintenant l'entrefer constant le long de la surface d'entrefer revient à augmenter la rigidité mécanique de la partie mobile et par voie de conséquence le diamètre de celle-ci. Or, dans une architecture polyentrefers, la multiplication des parties mobiles actives nécessite, à volume donné, au contraire de réduire au maximum les dimensions de des parties mobiles afin de pouvoir disposer d'un grand nombre de surfaces d'entrefers.

Une des solutions est donc de disposer d'un guidage mécanique «réparti », c'est-à-dire un guidage par frottement (ou éventuellement roulant) directement sur la partie active (voir figure 13b). 


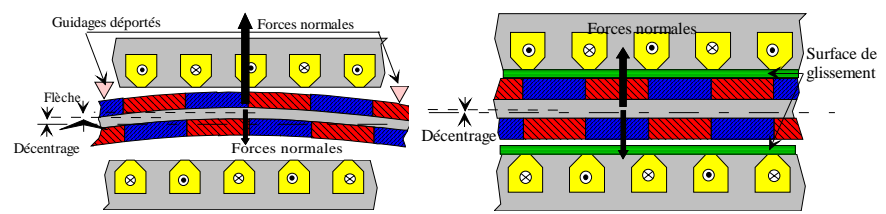

(a) guidage ponctuel classique Fig. 13 Structures de guidage

(b) guidage par bande de glissement

Le décentrage est alors au maximum égal au jeu mécanique résiduel qui, lui-même, est dû aux tolérances d'usinage et de montage. La valeur de l'effort normal est donc directement liée à ce dernier.

D’un point de vue mécanique, il n'est plus nécessaire de compenser mécaniquement les efforts normaux $\mathrm{F}_{\mathrm{n}}$. Mais, les forces de frottement sont alors directement proportionnelles aux efforts normaux : $F_{f}=\alpha \cdot F_{n}$, avec $\alpha$ le coefficient de frottement entre la tige et la surface de frottement.

Il faut donc des surfaces de glissement à très faible coefficient de frottement. La nature de ces surfaces peut être soit magnétique soit amagnétique. Cette technique permet d'envisager des entrefers extrêmement fins puisqu'il n'ont plus à prendre en compte la flèche éventuelle de la partie mobile. Seules les tolérances mécaniques de réalisation sont à prendre en compte

Sur la figure 14, nous avons représenté l'effort normal calculé dans le cas d'un guidage par bande de la figure 13b [25].

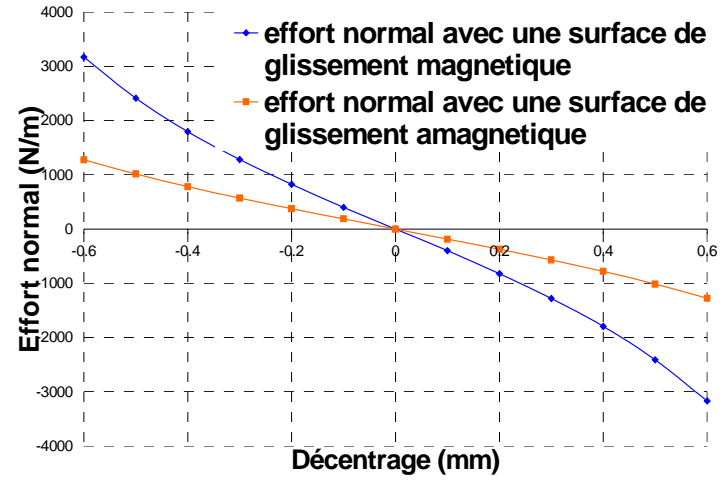

Fig. 14 Evolution de l'effort normal en fonction du décentrage pour des structures frottantes et un jeu mécanique total de $2 \mathrm{~mm}$ [25].

Ce résultat montre que le cas de surface amagnétique est favorable à la diminution de l'effort normal. Elle engendre cependant une réduction du flux inducteur maximal (donc de l'effort moyen) d'environ $15 \%$ par rapport à la solution utilisant une surface magnétique.

\section{La transmission de l'effort}

Si on s'intéresse au fonctionnement mécanique, chaque tige peut être modélisée par une liaison glissière. Les axes de ces liaisons ne peuvent pas être exactement parallèles (défaut d'usinage et de montage). Si ces tiges sont rigidement liées les unes aux autres, ce système est hyperstatique.

D'autre part, étant donné le faible diamètre des tiges d'une structure à très haut niveau de fractionnement, celles-ci ne peuvent fonctionner qu'en traction. Sinon, elles risqueraient un flambage mécanique.

La solution retenue consiste à transmettre l'effort via un arbre central. Les tiges sont solidaires de celui-ci à travers une liaison point-plan, comme représenté sur le schéma cidessous. Notons que sur ces figures, les jeux mécaniques entre les tiges et la pièce de liaison sont exagérés pour des raisons de facilité de compréhension.

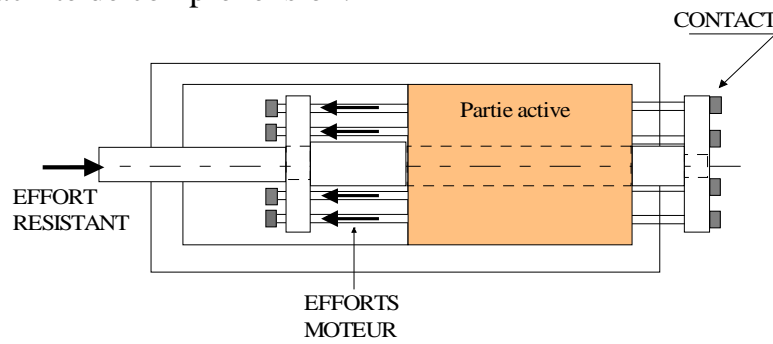

(a)

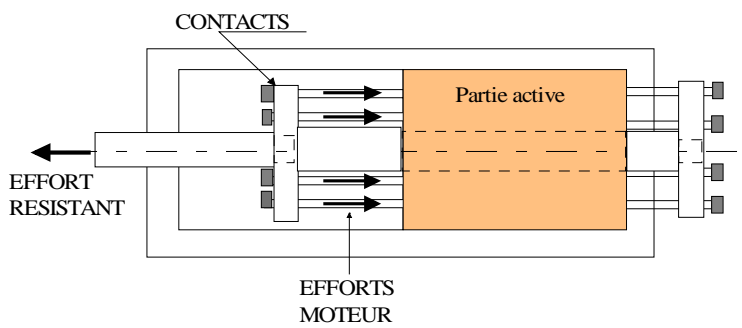

(b)

Fig. 15 Principe de transmission de l'effort pour un fonctionnement dans les deux sens (pour la compréhension, les jeux mécaniques entre les tiges et la pièce de liaison sont ici exagérés ).

Cette solution permet de ne faire travailler les tiges mobiles qu'en traction. Elles peuvent donc être prétendues, et ainsi atteindre des diamètres beaucoup plus faibles que pour une structure devant transmettre l'effort par poussée ce qui favorise la réduction des diamètres des tiges et donc de disposer d'un grand nombre de tiges.

\section{Le capteur de position intégré}

Dans l'optique de l'autopilotage de l'actionneur, un capteur de position a été conçu et intégré à l'actionneur. Ce capteur est basé sur le principe de la réluctance variable. C’est un circuit magnétique constitué d'une partie fixe et d'une partie mobile, ici l'une des tiges de l'actionneur polyentrefers. Ce capteur a été conçu pour que la position de la tige change la réluctance du circuit avec la même période que celle de conversion d'énergie.

Nous avons choisi deux bobinages indépendants entourant ce circuit magnétique. Le premier, bobinage d'excitation, est alimenté à haute fréquence par un courant alternatif. Il génère un champ alternatif dans le circuit magnétique. Le flux embrassé par le bobinage secondaire dépend de la position du capteur. Un traitement du signal, adapté sur la tension mesurée à ses bornes, nous donne une image de la réluctance du circuit magnétique, réluctance directement liée à la position de la tige.

Dans notre actionneur polyentrefer, la partie mobile est composée de tiges cylindriques de faible diamètre. Ces tiges 
sont constituées d'une alternance de rondelles magnétiques et amagnétiques.

Certaines de ces tiges constituent donc également la partie mobile des capteurs de position. Les parties fixes de ces capteurs sont constituées d'anneaux magnétiques (structures en deux coupelles), remplis par les deux bobinages solénoïdaux d'excitation et de mesure (figure 16).

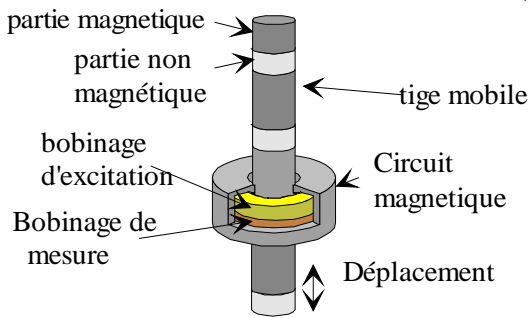

Fig. 16 Principe des capteurs de position réalisés

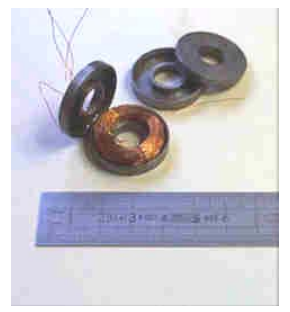

Afin d'obtenir directement un signal d'autopilotage pour chaque phase, nous avons choisi d'utiliser trois capteurs.

Toutes les tiges de l'actionneur sont mécaniquement synchrones. Pour des raisons d'encombrement axial, chaque anneau est placé autour d'une tige différente (figure 17). Le décalage entre la position de chaque capteur doit être d'un tiers du pas (modulo le pas), ce qui créé le déphasage électrique nécessaire entre les trois signaux de sortie. capteurs de position

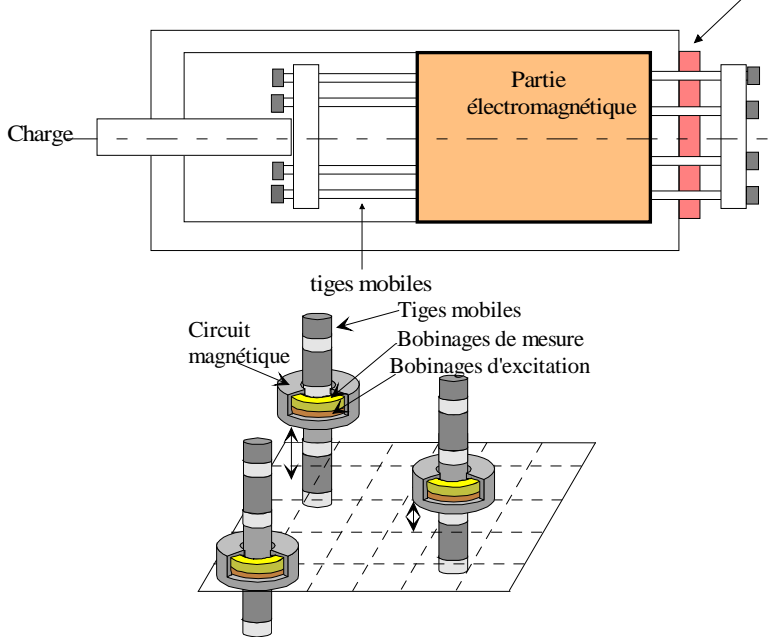

Fig. 17 Position des capteurs

Sur la figure ci-dessous est représenté à titre d'exemple la variation calculée de l'inductance du bobinage d'excitation du capteur (primaire) en fonction de la position de la partie mobile (tige).

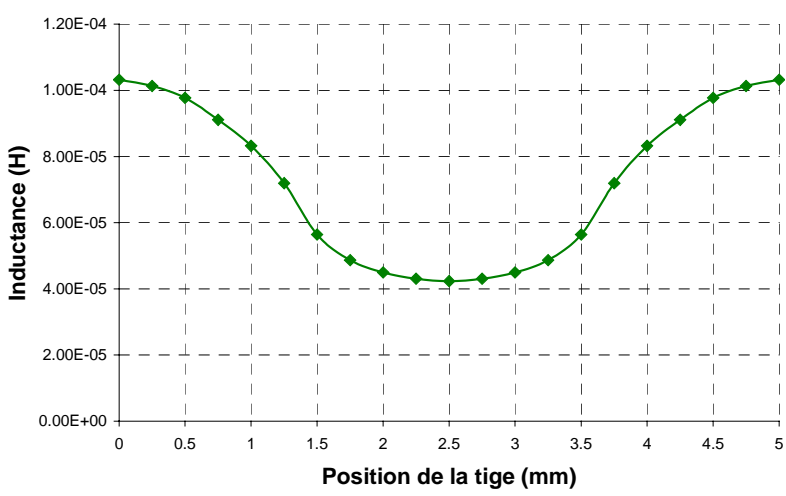

Fig. 18 Inductance calculée du bobinage primaire du capteur en fonction de la position

Grâce à un un conditionnement analogique ou numérique adéquat des signaux de sortie des capteurs (démodulation), nous pouvons déduire aisément la position de la partie mobile et ainsi réaliser l'autopilotage de l'actionneur [26].

\section{Performances theoriques de L’Actionneur MULTI-TIGES}

\section{A. Méthodologie d'optimisation}

L'optimisation globale des actionneurs à bobinage centralisé s'effectue d'une manière générale sur deux niveaux de modélisation. Le premier est lié aux dimensions locales correspondant à celles du motif élémentaire. A cette échelle, le calcul est réalisé par éléments finis non-linéaire axisymétrique.

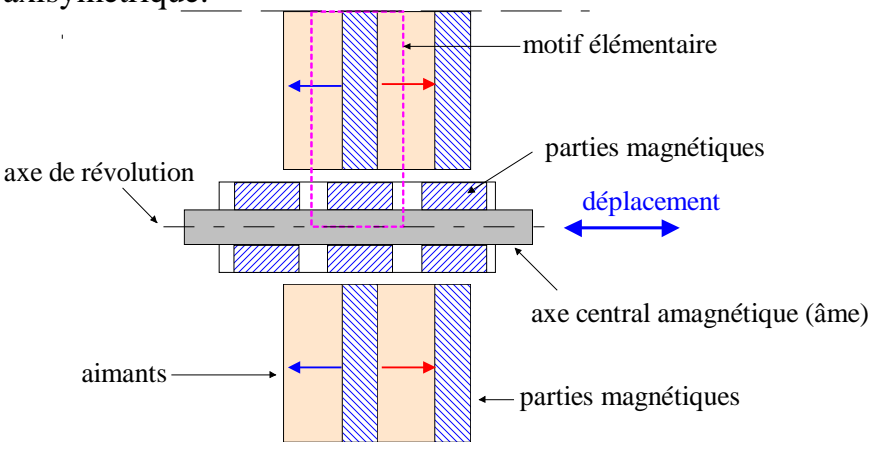

Fig. 19 Motifs élémentaire pour l'optimisation des actionneurs multi-tiges

Le second niveau est lié aux dimensions globale de l'actionneur correspondant à l'échelle du bobinage et des circuits de retour. Dans ce cas, la modélisation est effectuée par un schéma réluctant [3].

D’autre part, pour simplifier l'étude, nous considérons une alimentation en créneaux de courant. Ceci permet d'une part d'estimer les performances maximales de l'actionneur et d'autre part permet de simplifier les calculs de la force. En effet, dans ce mode d'alimentation, la force moyenne est directement liée à la seule caractéristique flux/ampères-tours de conjonction. Une seule position est donc à considérer. On utilise également la symétrie cylindrique des motifs élémentaires pour minimiser le temps de calcul. 
Les contraintes mécaniques de réalisation, de montage et de guidage utilisées dans la procédure d'optimisation sont les suivantes :

- le rayon de l'âme centrale $\mathrm{d}_{0} / 2$ (3 valeurs : $0 \mathrm{~mm}$, 4,6 mm, même valeur du prototype : 2,3 mm ) ;

- l'entrefer e $(0,1 \mathrm{~mm}$, valeur pour le prototype, 0,2 $\mathrm{mm}$ et $0,31 \mathrm{~mm}$ ) ;

- le pas dentaire $\tau$ (maintenu constant à $5 \mathrm{~mm}$, même valeur pour prototype, correspondant à la valeur optimale obtenue pour un entrefer de $0.1 \mathrm{~mm}$ ) ;

- les dimensions extérieures de l'actionneur (identiques à celles de notre prototype, présentant un volume actif de 1 $\left.\mathrm{dm}^{3}\right)$.

Les paramètres à optimiser (voir figure 20) sont alors :

(b) ;

- la largeur de la partie ferromagnétique de la tige

- le diamètre de la tige $(\mathrm{d})$;

- le diamètre des aimants ( $\mathrm{D})$;

- la largeur des aimants (a ) ;

- la section bobinable ;

- l'épaisseur des circuits de retour.

Les contraintes thermiques sont prises en compte à travers un modèle simplifié. Ce modèle moyen analytique, valable en régime permanent thermique, considère un refroidissement surfacique par convection :

pertes $=\alpha_{\mathrm{th}} \cdot \mathrm{S}_{\mathrm{th}} \cdot \Delta \mathrm{T}$

où $\alpha_{\text {th }}$ est le coefficient d'échange thermique $\left(10 \mathrm{~W} / \mathrm{m}^{2 \circ} \mathrm{K}\right.$ pour une convection naturelle), $S_{\text {th }}$ est la surface d'échange thermique correspondant à la surface extérieure de l'actionneur. Il a été également pris en compte la conduction thermique dans les isolants électriques entre le bobinage et la culasse extérieure. Enfin, $\Delta \mathrm{T}$ est l'élévation de température maximale admissible.

Concernant les pertes, nous n'avons considérés que les pertes Joule, les pertes magnétiques et mécaniques ont été négligées dans ce modèle thermique.

D'autre part, la caractéristique $\mathrm{B}(\mathrm{H})$ des circuits magnétiques est non-linéaire. La courbe $\mathrm{B}(\mathrm{H})$ considérée présente un coude de saturation aux alentours de $1.6 \mathrm{~T}$.

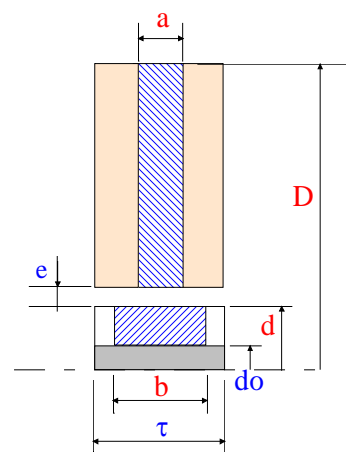

Fig. 20 Paramètre du motif élémentaire. Les contraintes mécaniques sont représentées en bleu. Les paramètres à optimiser sont en rouge
Enfin, d'un point de vue topologique, les tiges sont réparties en couches successives centrées sur l'axe de l'actionneur comme illustré par la figure 21.

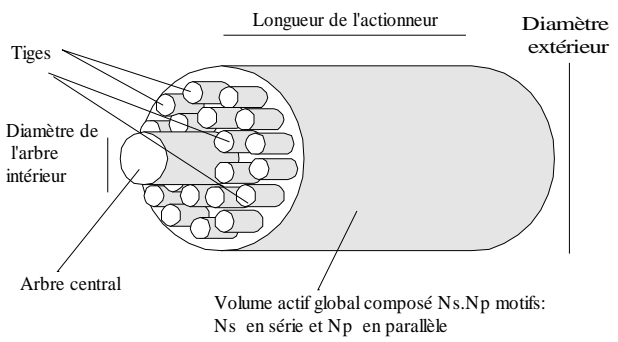

Fig. 21 Répartition des tiges

Un algorithme génétique permet d'optimiser les différents paramètres simultanément. Il utilise des populations de 400 individus pour environ 400 générations.

\section{B. Résultats}

Les résultats de ces optimisations peuvent être résumés dans les abaques des figures 22 et 23 correspondant respectivement à l'influence des deux principales contraintes mécaniques : le diamètre de l’âme centrale des tiges et l'entrefer [25].

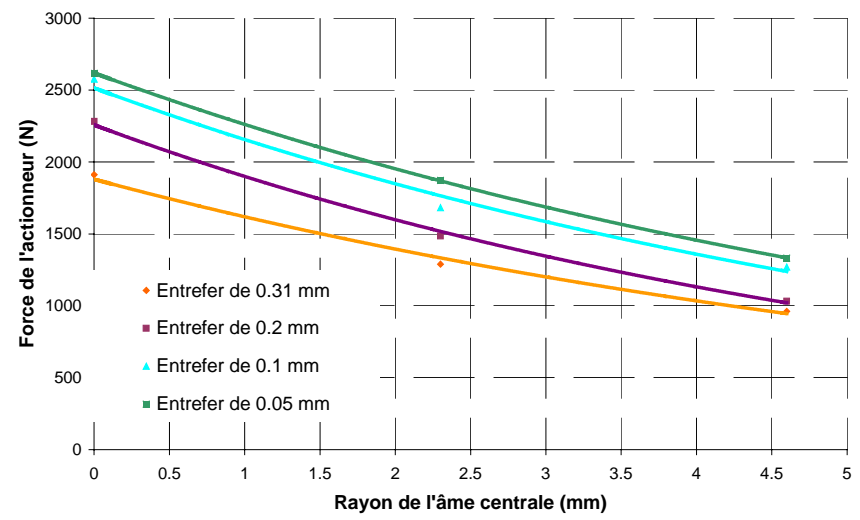

Fig. 23 Evolution théorique de la force de l'actionneur en fonction du rayon intérieur des tiges (pour un volume actif de $1 \mathrm{dm}^{3}$ et un pas de $5 \mathrm{~mm}$ )

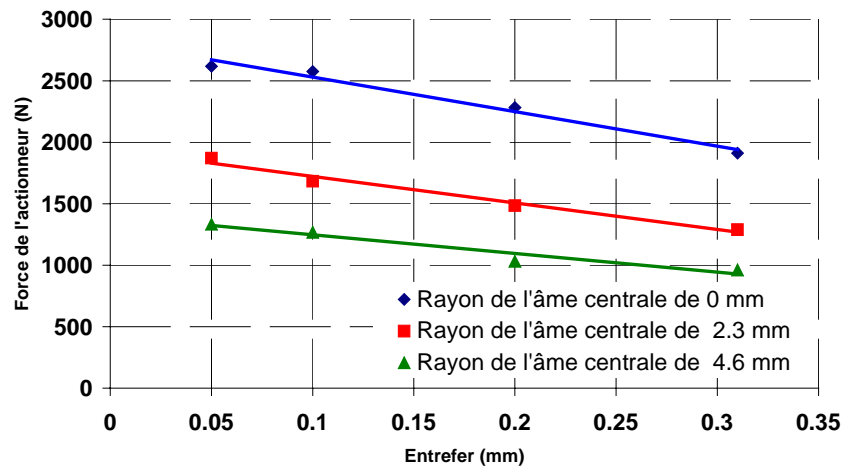

Fig. 24 Evolution théorique de la force de l'actionneur en fonction de l'entrefer (pour un volume actif de $1 \mathrm{dm}^{3}$ et un pas de $5 \mathrm{~mm}$ )

L'évolution du nombre de tiges optimal est donnée à la figure 25. 


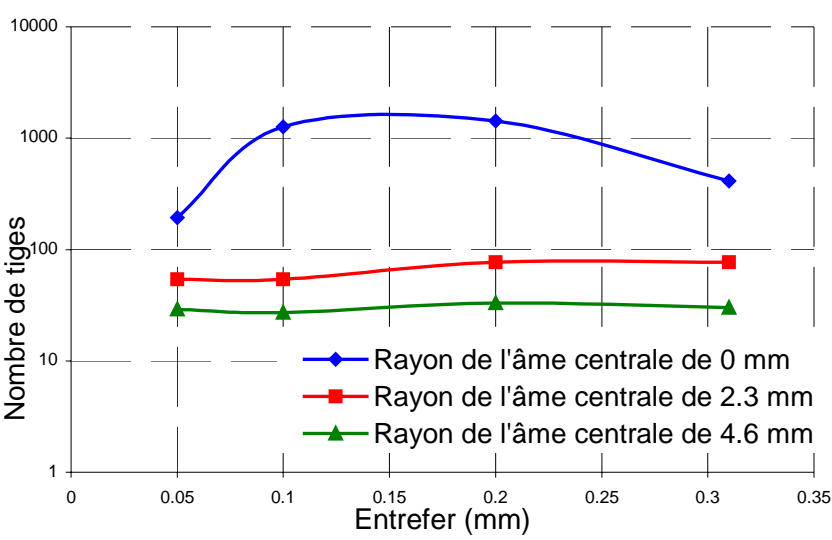

Fig. 25 Evolution théorique du nombre de tiges optimal en fonction de l'entrefer (pour un volume actif de $1 \mathrm{dm}^{3}$ et un pas de $5 \mathrm{~mm}$ ).

Plus le rayon de l'âme centrale des tiges est faible, plus les dimensions du motif optimal sont faibles. On a donc plus de tiges. On voit donc que le nombre de tiges des actionneurs optimisés est très important.

Ces optimisations sous contraintes permettent d'estimer rapidement dans le cas général les performances des actionneurs multi-tiges. En effet, par rapport à un cas de référence (cas considéré précédemment: pas $\tau^{*}=5 \mathrm{~mm}$, volume actif $\mathrm{V}^{*}=1 \mathrm{dm}^{3}$, coefficient d'échange thermique $\alpha_{\mathrm{th}}{ }^{*}=10 \mathrm{~W} / \mathrm{m}^{2}{ }^{\circ} \mathrm{K}$ et $\Delta \mathrm{T}=100^{\circ} \mathrm{K}$ ), nous pouvons en déduire les performances d'un actionneur multi-tiges de caractéristiques différentes à partir des lois de similitude :

$\mathrm{F}=\mathrm{F}^{*}\left(\frac{\mathrm{V}}{\mathrm{V}^{*}}\right)^{7 / 6} \cdot\left(\frac{\alpha_{\mathrm{th}} \cdot \Delta \mathrm{T}}{\alpha_{\mathrm{th}}{ }^{*} \cdot \Delta \mathrm{T}^{*}}\right)^{0.5} \cdot\left(\frac{\mathrm{e}}{\mathrm{e}^{*}}\right)$

où $\mathrm{F}^{*}$ est la poussée obtenue pour le cas de référence.

\section{LE PROTOTYPE}

\section{A. Caractéristiques et éléments de réalisation}

Un premier prototype a été réalisé au laboratoire dont la principale fonction était de valider les solutions mécaniques adoptées et sa faisabilité pré-industrielle. Les principales dimensions du prototype sont résumées dans le tableau suivant :

\begin{tabular}{lc}
\multicolumn{2}{c}{ TABLE 1 DIMENSIONS PRINCIPALES DU PROTOTYPE } \\
\hline Désignations & valeurs \\
\hline Entrefer $(\mathrm{mm})$ & 0,1 \\
\hline Diamètre intérieur des tiges $(\mathrm{mm})$ & 4,6 \\
\hline Diamètre extérieur des tiges $(\mathrm{mm})$ & 10 \\
\hline Pas $(\mathrm{mm})$ & 5 \\
\hline Epaisseur des aimants $(\mathrm{mm})$ & 1,5 \\
\hline Nombre de tiges & 8 \\
\hline Nombre de motifs en série & 8 \\
\hline Diamètre de la partie active $(\mathrm{mm})$ & 85 \\
\hline Longueur totale de l'actionneur $(\mathrm{mm})$ & 110 \\
\hline Diamètre extérieur de l'actionneur $(\mathrm{mm})$ & 122 \\
\hline Section du conducteur $\left(\mathrm{mm}^{2}\right)$ & 1 \\
\hline Nombre de spires & 160 \\
\hline Résistance de chaque phase $(\Omega)$ & 1.4 \\
\hline
\end{tabular}

a) La partie active fixe

La partie active fixe est composée de trois phases comme le montre la figure 25. Les motifs élémentaires sont constitués d'un empilement de plaques entre lesquelles viennent s'intercaler les aimants aimantés axialement (voir figure 26).
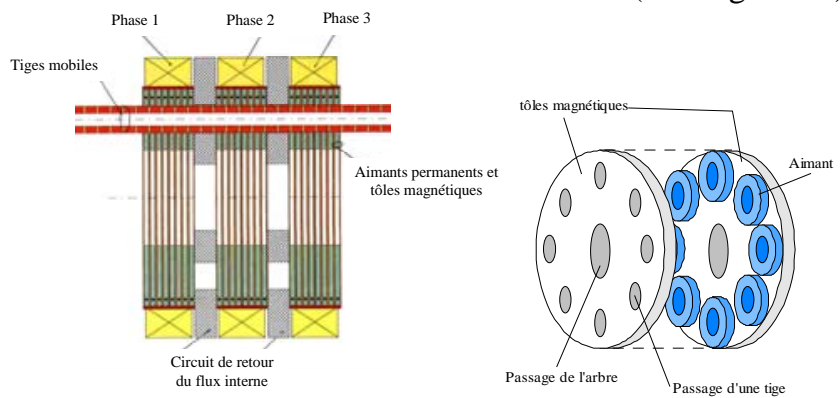

Fig. 25 Détails de la partie électromagnétique

Les aimants sont de type NdFeB alliés ayant une induction rémanente de $0,65 \mathrm{~T}$.

De leur coté, les tôles FeSi3\% ont été réalisées par découpe laser. Elles sont percées notamment de 8 trous d'un diamètre de 10,2 mm, destinés à recevoir les 8 tiges (figure 26).
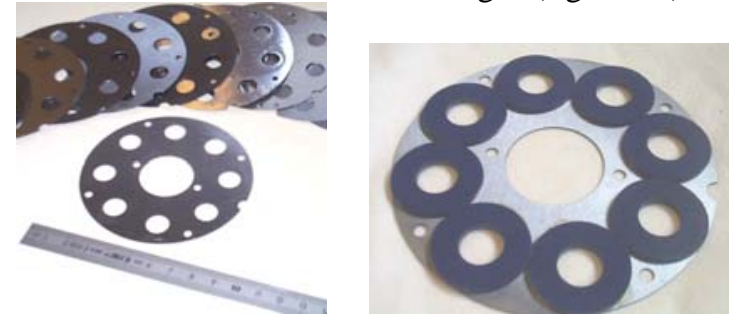

Fig. 26 Plaques composites (tôles et aimants)

b) Les parties actives mobiles (tiges)

Les tiges sont constituées d'un empilement de bagues magnétiques et amagnétiques placées sur une âme amagnétique. Elles ont ensuite été rectifiées afin d'obtenir un diamètre de $10 \mathrm{~mm}$ et un état de surface régulière (voir figure 27).

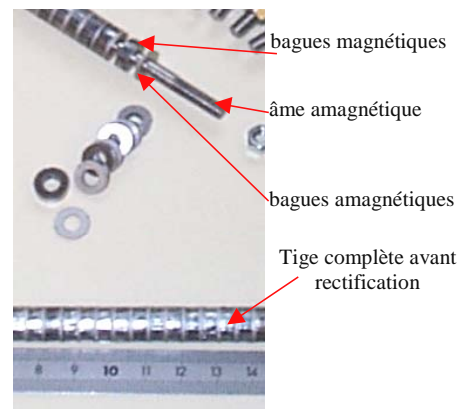

Fig. 27 Tiges composites de l'actionneur

La méthode de guidage glissant décrite précédemment a été appliquée. La solution est de guider chaque tige indépendamment sur une surface de glissement dans l'entrefer (voir figure 28). Cette surface de glissement doit avoir une épaisseur très légèrement inférieure à celle de l'entrefer afin de tenir compte des imperfections du montage du stator. 


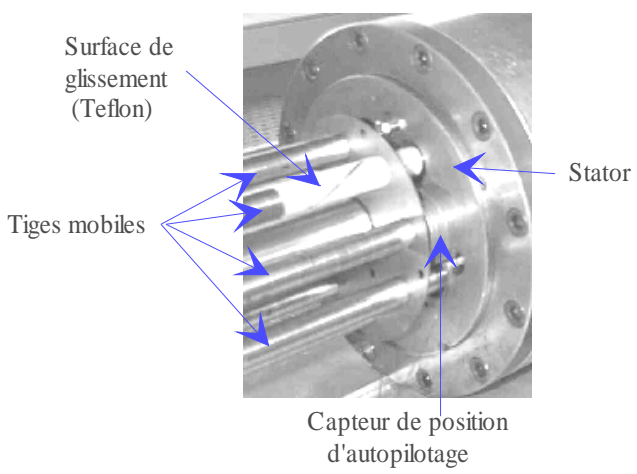

Fig. 28 -Photo des guidages glissants de l'actionneur multi-tiges

Les pertes mécaniques sont minimisées par l'utilisation d'un matériau (le Teflon) ayant un très faible coefficient de frottement $\alpha$ avec les tiges. Ici, ce coefficient est de l'ordre de 0.1 . Ce système reste à l'état artisanal et nécessiterait d'être amélioré pour passer au stade industriel.

Les deux parties actives fixe et mobile montées sont représentée sur la figure ci-dessous où le système de transmission de la force (culasse extérieure amagnétique) n’est pas représentée.

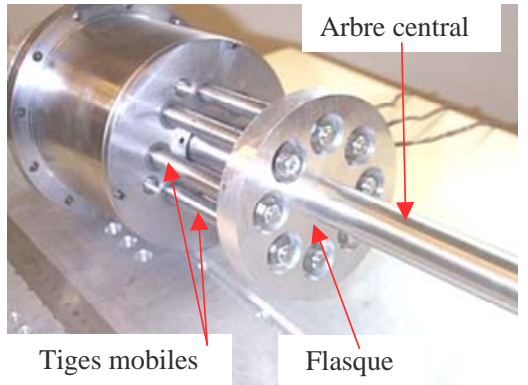

Fig. 29 Photo des guidages glissants de l'actionneur multi-tiges

\section{B. Mesures statiques}

Les mesures statiques ont été réalisées sur le principe de la mesure de la force de décrochage. Les bobinages de puissance étant alimentés par une source de courant continu et montés comme indiqué sur la figure 30.
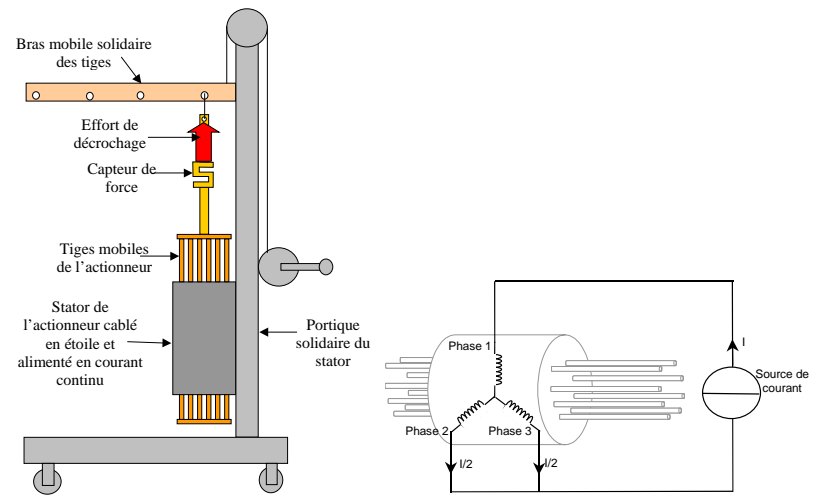

Fig.30 Banc d'essai de décrochage (statique)

Ces essais ont permis de mesurer la poussée générée pour différentes valeurs de courant d'induit. Ces résultats, conformes aux calculs, sont donnés à la figure 31 .

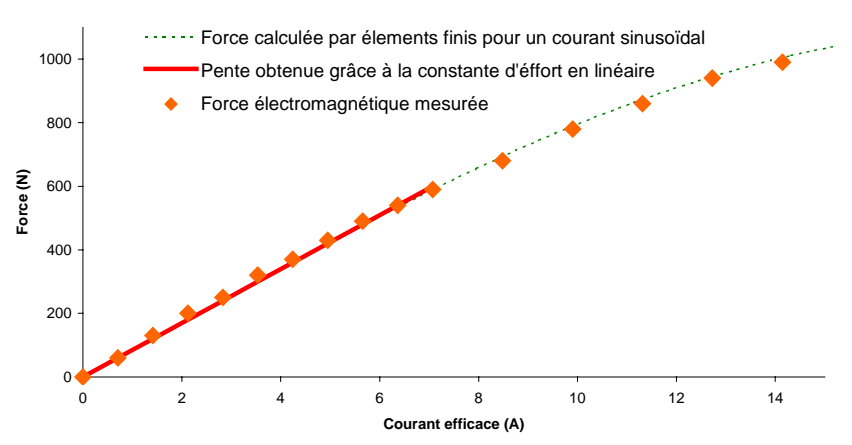

Fig. 31 Comparaison entre la force à basse vitesse simulée par éléments finis et l'effort électromagnétique mesuré

Ainsi, le volume total (hors course) de cet actionneur étant aux alentours de $1 \mathrm{dm}^{3}$, la force volumique atteinte dans des conditions normales de refroidissement est de $1000 \mathrm{~N} / \mathrm{dm}^{3}$.

\section{Mesures dynamiques}

Un banc instrumenté a été réalisé. Celui-ci (voir figure 33) est composé de l'actionneur décrit précédemment, d'un capteur de force, d'une charge inertielle réglable, et d'un capteur de position. L'alimentation de l'actionneur et son autopilotage sont effectués classiquement à partir d'un onduleur de tension triphasé piloté en courant à partir d'une carte DSP.

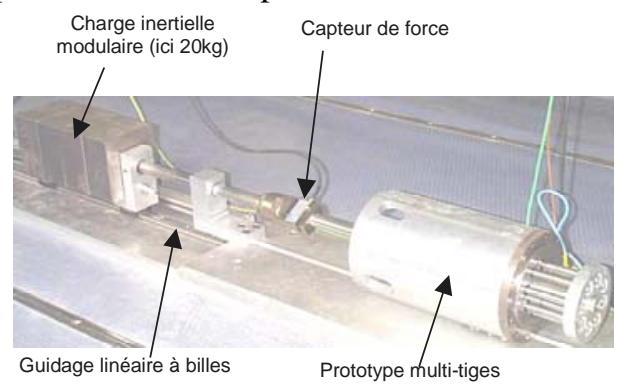

Fig. 32 Banc d'essai dynamique

Nous avons tout d'abord effectué des essais dynamiques à vide afin de valider la stratégie d'autopilotage et le bon fonctionnement du banc. Nous présentons à la figure 33 la réponse en position obtenue pour une consigne de position en échelon de $4 \mathrm{~cm}$. La vitesse maximale atteinte pour un courant crête de 15 A est d'environs $0.2 \mathrm{~m} / \mathrm{s}$.

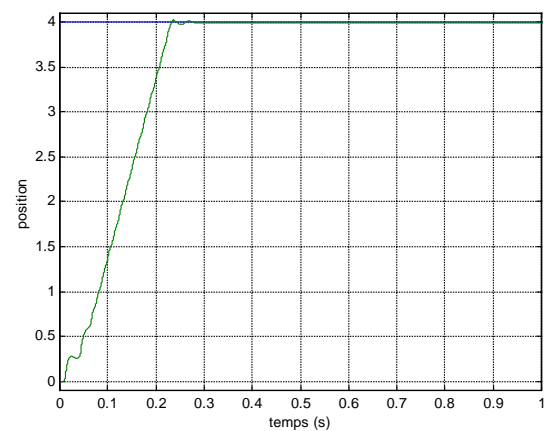

Fig. 33 Réponse (vert) à un échelon de position (bleu) avec une limitation à 15 A (position en $\mathrm{cm}$, temps en s)

Ensuite, des essais en charge ont été réalisés. A la figure 34 est présentée la réponse à une consigne en rampe de position pour une charge de $20 \mathrm{Kg}$ et pour le même courant maximal. 
La vitesse maximale atteinte tout en ayant un fonctionnement satisfaisant est de $0.16 \mathrm{~m} / \mathrm{s}$. D'autre part, la réponse est beaucoup plus lisse que lors des essais à vide du fait de l'atténuation des oscillations à travers l'inertie de la charge.

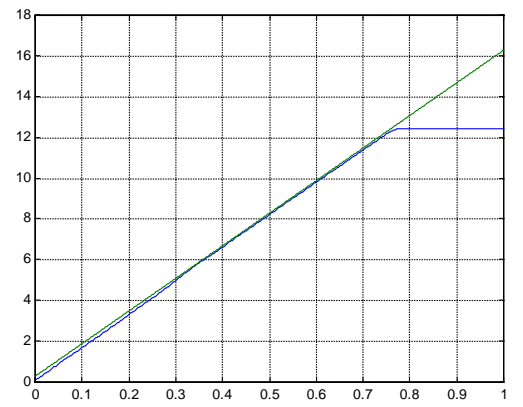

Fig. 34 Réponse (bleu) à une rampe de position (bleu) pour une charge de 20 $\mathrm{kg}$ et un courant maximal de $15 \mathrm{~A}$ (position en cm, temps en s)

\section{CONCLUSION}

Cet article traite de la conception des actionneurs polyentrefers à bobinages globaux. Suivant ce concept, nous avons étudié et réalisé une structure originale d'actionneur linéaire à aimants permanents multi-tiges. L'expérience acquise lors des précédentes réalisations nous a incité à prendre en compte dès la conception les problèmes essentiellement mécaniques liés à ce type d'architecture. Des solutions innovantes ont été développées et ont permis des résoudre de nombreux problèmes. Cependant, quelques difficultés restent à surmonter. En premier lieu, le guidage mécanique. En effet, nous avons utilisé pour cela du Téflon qui s'avère être un bon matériau en terme de coefficient de frottement mais présente de mauvaises qualités en termes de tenue mécanique et thermique. Nous envisageons de le remplacer par une bande de bronze fine rectifiée après montage. Un autre problème est lié au circuit magnétique de retour. Celui-ci est aujourd'hui massif. Ceci limite les dynamiques mécaniques (fortes accélérations). L’obtention de dynamiques élevées requerrait l'utilisation de matériau isotrope (car flux 3D) à faible pertes magnétiques, tel que la poudre de fer. Enfin, aspect non développé dans cet article, le facteur de puissance de ces dispositifs optimisés sur le seul critère de densité de force reste faible et sans doute insuffisant pour des applications à vitesses élevées.

\section{BIBLIOGRAPHIES}

[1] C. Rioux, " Aspects préliminaires de la théorie des machines électriques comportant des matériaux ferromagnétiques », Revue de Physique Appliquée, $\mathrm{N}^{\circ} 15,1980$, pp. 1505-1515.

[2] P.E. Cavarec, H. Ben Ahmed, B. Multon "Force Density Improvements from Increasing the Number of Airgap Surfaces in Synchronous Linear Actuators", IEE Proceedings, Electric power application Volume 150, Issue 01. January 2003, pp.106-120.

[3] H.Ben Ahmed, L.Prévond, B.Multon, «Structures polyentrefers : solutions pour les entraînements directs ? », Colloque SEE, CEMD’99, Cachan, 4 février 1999, pp. 95-100.

[4] P.Letellier «Les entraînements directs de forte puissance et à basse vitesses », CEMD’99 La conversion électromécanique directe, 4 février 1999 ENS Cachan SEE P 22-30.

[5] B. Multon, J. Bonal «Les entraînements électromécaniques directs : diversité, contraintes et solutions. », Revue REE $n^{\circ} 10$, novembre 99, pp.67-80.
[6] D.Matt, « Etude de deux structures originales de machine à réluctance variable polyentrefer », thèse de doctorat, Paris 6, avril 1987

[7] P.Bolognesi, L.Tapanecco "Analysis of an unconventional linear PMAC machine suitable for reciprocating motion generation systems ", Electromotion 2001 Bologngna (italy), june 19-20,2001 P 443-448.

[8] P.C.Coles et Al. " Propeller drive motors for high altitude aircraft », Electromotion 2001 Bologngna (italy), june 19-20,2001 pp.391-394.

[9] Uday S.Deshpande et Al. "High-force density linear switched Reluctance Machine », IEEE Transactions on industry applications, Vol31, N², March/April 1995 pp.345-352.

[10] H.Weh «Synchronous machines with new topologies », SM100 27-28 august 1991 Zurich.

[11] J.P.Bastos, R.Goyet, J.Lucidarme, «Performances intrinsèques des machines à réluctances variables à disques imbriqués », revue de physique appliquée $\mathrm{n}^{\circ} 1$ pp.45-53, janvier 1985.

[12] Lewis E.Unnewehr « Magnetic circuit studies for an axial-gap reluctance motor ", Electric machines and Electromechanics: An International Quarterly, 1 :57-69, 1976.

[13] D. Matt, R. Goyet, J. Lucidarme and C. Rioux, "Longitudinal Field Multi-Airgap Linear Reluctance Actuator", Electric Machines \& Power Systems, 1987, 13:299-313.

[14] J. Lucidarme, A. Amouri, M. Poloudjadoff : «Optimum design of Longitudinal Field Variable Reluctance Motors - Application to high Performance Actuator », IEEE Trans. On Energy Conversion, vol. 8, $\mathrm{N}^{\circ}$.3, September 1993

[15] H.Ben Ahmed, « Contribution à la conception des machines synchrones à aimants permanents et bobinage global », thèse de doctorat , Paris VI, janvier 1994.

[16] J.Lucidarme, C.Rioux, J.Pouillange, «Moteur discoïde à réluctance variable et à aimants permanents. Des couples spécifiques élevés à faible vitesse », RGE n³ pp.43-52, mars 1987.

[17] P.F.Desesquelles, J. Lucidarme, H.Ben Ahmed « Expérimentation d'une machine couple à très hautes performances massiques ", Colloque M.O.P.P., Lausanne (Suisse) Juillet 1990.

[18] Poulot "Contribution à l'étude des machines à réluctance variable et à aimants. Structure à concentration de flux », Thèse de doctorat, Institut national polytechnique de Toulouse, Novembre 1995.

[19] M.Amiet, J.Lucidarme Brevet n 9515707, dépôt le 29/12/1995, au nom de l'Etat français.

[20] H. Ben Ahmed, L. Prévond, B. Multon, B. Salamand and J. Lucidarme, "Special Synchronous Linear Actuators: Structures and Performances", Revue Electromotion, n5, 1998, pp. 93-101.

[21] H.Ben Ahmed, P.E.Cavarec, L.Prévond, B.Multon « Conception et étude d'un actionneur linéaire synchrone à aimants permanents et multiplaques », Electrotechnique du Futur 14-15 novembre 2001 Nancy pp.307-312.

[22] H.Ben Ahmed, B.Multon, P.E.Cavarec, M. Antunes-Menoita « Actionneur ou générateur linéaire à tiges », Brevet $\mathrm{N}^{\circ} 0208194$, dépôt le $1^{\text {er }}$ juillet 2002, déposant CNRS.

[23] M.Antunes-Menoita «Modélisation, conception et réalisation d'un actionneur linéaire multi-tiges à haute densité d'efforts », Mémoire CNAM en électrotechnique soutenu le 29 novembre 1999.

[24] P.E. Cavarec :"Conception, modélisation et optimisation des actionneurs polyentrefers à bobinages globaux : application aux structures linéaires multi-tiges ", thèse de doctorat, ENS Cachan, novembre 2002.

[25] P.E.Cavarec, H.Ben Ahmed, B.Multon «New multi-rod linear actuator for direct-drive, wide mechanical band pass applications », IEEE IAS annual metting 2002 Pittsburg, Pennsylvania USA October 13-18, 200208-21.

[26] P.E.Cavarec, H.Ben Ahmed, B.Multon « Integrated reluctance position sensor for the self-commutation of a hybrid linear electric actuator ", Recueil en CDRom de la conférence EPE 2001, Graz (Autriche) Septembre 2001.

[27] M.Bork, G.Henneberger « New Transverse Flux Concept for an Vehicle Drive System », ICEM 96, Vigo sept. 96, Vol. 2, pp.308-313.

[28] G.Henneberger, W.Evers, H.Elschenbroich «A transverse flux linear synchronous motor with a passive track », ICEM 2000.

[29] C. Chillet, J.P. Yonnet, P. Brissonneau « Machines synchrones à aimants permanents : structures à entrefer plan », RGE $N^{\circ}$ 6, juin 1990.pp. 8-10. 